\title{
Ökonomische Auswirkungen des Zusammenspiels von Managern und Betriebsräten
}

\author{
Annette van den Berg \\ Yolanda Grift \\ Arjen van Witteloostuijn
}

Wie Betriebsräte die Effizienz eines Unternehmens beeinflussen, wird in der Literatur über industrielle Beziehungen ausführlich diskutiert. Sträflich vernachlässigt wird allerdings zumeist die Frage, wie sich das Zusammenspiel zwischen Management und Interessenvertretung auf den Erfolg einer Organisation auswirkt. Mit Rückgriff auf die Daten einer niederländischen Befragung von Unternehmensleitungen aus der Privatwirtschaft soll diese Lücke gefüllt werden. Sie zeigen, dass die Auswirkungen, die die Existenz eines Betriebsrats auf den Unternehmenserfolg hat, sowohl vom Verhalten des Managements gegenüber dem Betriebsrat beeinflusst werden als auch von der jeweiligen ökonomischen Situation des Betriebes, in der der Betriebsrat agiert. ${ }^{1}$

\section{Einleitung}

Wie in Literaturstudien beispielsweise von Addison et al. (2004) sowie Addison (2005) herausgearbeitet wird, sind die Befunde uneindeutig, welche wirtschaftlichen Effekte die Existenz einer Interessenvertretung hat. Unter Verwendung einer finanziellen Erfolgsgröße als Maßstab berichten beispielsweise einige Studien über positive Wirkungen (Addison et al. 2001), andere konstatieren einen negativen Einfluss (Dilger 2002), wieder andere finden keinen $\mathrm{Zu}$ sammenhang zwischen beiden Größen (Addison et al. 2004). Uneinheitlich sind die Ergebnisse auch, wenn andere Aspekte wie z. B. Innovation, Lohnhöhe oder Fluktuation hinzugezogen werden. Das bedeutet, dass weitere Untersuchungen durchgeführt werden müssen, um die zugrundeliegenden Zusammenhänge zwischen der Unternehmensleistung und dem Wirken der Betriebsräte erklären zu können. Unsere Studie versucht, dazu einen Beitrag zu leisten, indem sie bisherige Analysen um einen neuen Blickwinkel ergänzt: Gefragt wird danach, wie die Haltung der Unternehmensleitung gegenüber der Interessenvertretung deren Agieren - zum Wohle oder zum Nachteil des Unternehmens prägt, wobei zwischen wirtschaftlich guten und schlechten Zeiten für den Betrieb unterschieden wird.

Der Bedarf an weiteren Analysen ergibt sich aus einigen Verzerrungen, die die vorhandene Literatur dominieren. Erstens richtet der weitaus größte Teil aller Untersuchungen den Fokus ausschließlich auf betriebliche Interessenvertretungen in Deutschland. Unser Artikel konzentriert sich auf ein anderes Land mit einer langjährigen Betriebsrätetradition: die Niederlande. Zweitens verwenden die meisten vorliegenden Studien, mit wenigen Ausnahmen, ziemlich grobschlächtige Messgrößen. Bei der Analyse der Auswirkungen von Mitbestimmungsinstitutionen auf den Unternehmenserfolg wird vielfach nur die Existenz bzw. das Fehlen eines Betriebsrates als unabhängige Variable berücksichtigt. Wir ergänzen ein feineres Maß für die Wahrnehmung der Betriebsratstätigkeit, indem wir die Haltungen der Unternehmensleitung gegenüber dem Betriebsrat in unserer Analyse berücksichtigen. Die Untersuchung von Bryson et al. (2006), die anhand einer britischen Stichprobe zu dem Schluss kommt, dass die Produktivität stark gesteigert wird, wenn das Management konstruktiv auf die Mitwirkung von Arbeitnehmern (in welcher Form auch immer) reagiert, bestätigt unsere Herangehensweise. Und drittens erforschen wir auch, wie Mitbestimmung die wirtschaftliche Lage einer Organisation in Krisenzeiten beeinflusst, wenn die Organisation infolge negativer externer Schocks Restrukturierungsmaßnahmen durchführt und Arbeitsplätze abbaut. Diese Untersuchungsperspektive resultiert aus der Überlegung, dass Mitbestimmung in guten Zeiten problemlos funktionieren mag, während sie in schlechten Zeiten, wenn ein Unternehmen umstrukturiert wird, ernsthaft auf die Probe gestellt wird.

Unsere Daten wurden im Rahmen einer 1998 landes- und branchenweit durchgeführten Umfrage erhoben. Die Urheber dieses Datensatzes haben ihre wichtigsten
Ergebnisse jedoch lediglich in Form deskriptiver Statistiken veröffentlicht (Looise/ Drucker 2003; Van het Kaar/Looise 1999). Nach unserer Kenntnis gibt es bisher keine Studie, in der der Einfluss niederländischer Betriebsräte auf den Unternehmenserfolg im Rahmen einer multivariaten Regressionsanalyse untersucht worden ist. Daher präsentieren wir im Folgenden die ersten

\footnotetext{
1 Wir danken Robbert van het Kaar und Jan Kees Looise für die Bereitstellung der Daten. Außerdem danken wir Rob Alessie, Wolter Hassink, Jacques Siegers und den Teilnehmern der WSI-AIAS Konferenz "New perspectives for employee's participation: decentralisation tendencies and effectiveness of works councils" (Düsseldorf, 15. Mai 2008) für ihre wertvollen Kommentare.
}

Annette van den Berg, Dr., Lecturer in Institutional Economics, Universität Utrecht. Arbeitsschwerpunkte: Industrielle Beziehungen, Corporate Governance. e-mail: J.E.vandenBerg@uu.nI Yolanda Grift, Dr., Ökonometrikerin, Asistenzprofessorin am Department of Econometrics, Universität Utrecht. Arbeitsschwerpunkte: Mikroökonomie, Betriebsräte, Lohnbildung. e-mail: Y.Grift@uu.nl Arjen van Witteloostuijn, Prof. Dr., Wirtschafts- und Sozialwissenschaftler, Universität Antwerpen, Universität Utrecht. Arbeitsschwerpunkte: Demografie, Diversity. e-mail: A.vanWitteloostuijn@uu.nl

Übersetzung aus dem Englischen von Katja Rietzler 
empirischen Ergebnisse einer solchen Analyse.

Insbesondere prüfen wir, welche Faktoren den entscheidenden Erklärungsbeitrag für die wirtschaftliche Situation von Unternehmen leisten. Im Fragebogen wurden die Befragten gebeten, die wirtschaftliche Lage ihrer Organisation auf einer Skala von stark bis schwach einzuordnen. Empirisch prüfen wir ein umfassendes Organisationsmodell, das nicht nur die üblichen ökonomischen erklärenden Variablen wie Firmenund Arbeitnehmereigenschaften in der ersten Stufe der Regressionsanalyse beinhaltet, sondern in einer zweiten Stufe auch Variablen einschließt, die die Haltung des Managements und der Betriebsräte zu ihrer Zusammenarbeit reflektieren. In der abschließenden dritten Stufe der Regressionsanalyse nehmen wir die Wirkung der Betriebsratstätigkeit auf die wirtschaftliche Lage bei Umstrukturierungsmaßnahmen unter die Lupe, indem wir Interaktionsterme hinzufügen. Unsere statistischen Analysen sind also hierarchisch aufgebaut. Sie beginnen mit einer Basisschätzung, ermitteln dann die Haupteffekte und fügen schließlich Interaktionsterme hinzu. Durch diese Vorgehensweise hoffen wir, zu entschlüsseln, welche unmerklichen, situationsspezifischen Einflussfaktoren die Wirkung der Betriebsratstätigkeit auf die Unternehmensleistung steuern.

Abschnitt 2 skizziert kurz die unserer Untersuchung zugrundeliegenden Ausgangsüberlegungen. Auf dieser Grundlage gelangen wir zu fünf zu überprüfenden Hypothesen, die vier Haupteffekte und eine Reihe von Interaktionseffekten betreffen (Abschnitt 3). Danach werden in Abschnitt 4 die Daten und die Untersuchungsmethode eingeführt. Die wichtigsten Ergebnisse unserer Schätzungen werden in Abschnitt 5 vorgestellt. Ein Fazit ziehen wir in Abschnitt 6.

\section{Ausgangsüberlegungen}

In den Niederlanden haben Betriebsräte genau wie in Deutschland weitreichende Befugnisse. Verbrieft sind ausreichende und rechtzeitige Informationsrechte, das Recht, an Beratungen teilzunehmen (im Hinblick auf bestimmte finanzielle und geschäftliche Angelegenheiten), oder auch, die Zustimmung zu verweigern (bei sozialen Belan- gen). Darüber hinaus sind sie berechtigt, externe Experten hinzuzuziehen, und sie können Gerichte anrufen, wenn sie ihre Rechte in irgendeiner Weise verletzt sehen.

Freeman/Lazear (1995) zeigen theoretisch, dass jedes dieser Rechte zum Vorteil aller Beteiligten genutzt werden kann, sodass sich eine „Win-Win“-Situation einstellt. Erstens kann eine gute Kommunikation zwischen der Leitung eines Unternehmens und den Arbeitnehmervertretern das Vertrauen im Unternehmen insgesamt stärken. Dies erweist sich insbesondere in wirtschaftlich schlechten Zeiten als relevant. Vertrauen kann dann dazu beitragen, Unruhe im Betrieb und ein Absinken der Arbeitsproduktivität zu vermeiden. Zweitens kann die Einbeziehung des Betriebsrats durch das Management helfen, organisatorische Engpässe zu überwinden. Drittens geben Mitbestimmungsrechte den Arbeitnehmern mehr Kontrolle über ihre eigenen Arbeitsbedingungen und über die Sicherheit ihrer Arbeitsplätze, was wiederum ihre Loyalität gegenüber dem Unternehmen befördert. Dadurch werden die Interessen von Arbeitnehmern und Anteilseignern besser in Einklang gebracht.

Als Gegenszenario diskutieren Freeman und Lazear auch die Möglichkeit, dass eine einflussreiche Belegschaft motiviert sein könnte, sich einseitige Vorteile zu verschaffen, beispielsweise durch höhere Lohnforderungen oder den Versuch, das Beschäftigungsniveau zu halten, selbst wenn dies letztlich die wirtschaftliche Lage des Unternehmens gefährdet. Was die Niederlande angeht, so schränkt das geltende Recht das Risiko eines solchen Rent-Seeking-Verhaltens seitens der Betriebsräte stark ein, weil es bei Lohnverhandlungen den Gewerkschaften als Tarifvertragspartei den Vorrang gibt. Jedoch könnten Betriebsräte die Funktionsfähigkeit eines Unternehmens negativ beeinflussen, wenn sie unzureichend informiert sind oder auch, weil sie Entscheidungsprozesse verlangsamen können, wenn sie glauben, dass dies in ihrem Interesse ist. Außerdem entstehen Kosten in Form von verlorener Arbeitszeit aufgrund von Sitzungen und Schulungen der Betriebsratsmitglieder, für Räumlichkeiten, die sie nutzen, und für das Engagement externer professioneller Berater. Sehr kostspielig ist es auch, wenn Betriebsräte ihr Recht wahrnehmen, vor Gericht zu ziehen.

Beeinflusst durch Freeman/Medoff(1984) betonen Bryson et al. (2006) die Bedeu- tung der Reaktion des Managements auf jegliche Form der Arbeitnehmermitsprache. Auf der Grundlage von empirischen Befunden für Privatunternehmen in Großbritannien ziehen sie den Schluss, dass das Funktionieren dieses „Mitsprache-Mechanismus“ („voice mechanism“ bzw. „voice practice“ bei Bryson et al. 2006) von der Haltung des Managements zur Mitbestimmung abhängt und dass folglich der Unternehmenserfolg in hohem Maße davon beeinflusst wird, inwieweit das Management geneigt ist, die Arbeitnehmer bei der Unternehmenspolitik mitreden zu lassen.

\section{Hypothesen}

Auf Basis dieser grundsätzlichen Ausgangsüberlegungen und vorhandener empirischer Befunde können wir nun mehrere Hypothesen entwickeln, die anschließend überprüft werden. Aus den bisherigen Ausführungen schlussfolgern wir, dass die Existenz von Mitbestimmung eine wichtige, aber keine hinreichende Bedingung für die Stärkung des - wie auch immer gemessenen - Unternehmenserfolgs ist. Sie hängt letztlich davon $a b$, in welchem Maße die Arbeitnehmervertreter von der Unternehmensleitung ernstgenommen werden und im Gegenzug davon, inwieweit Arbeitnehmer bereit und in der Lage sind, mit dem Management zusammenzuarbeiten, um Probleme zu lösen. Nur eine wechselseitige fruchtbare Zusammenarbeit kann einen positiven Beitrag zur wirtschaftlichen Lage und Entwicklung des Unternehmens leisten. Dies führt uns zu vier Hypothesen über die Haupteffekte.

(1) Je stärker das Management geneigt ist, eine offene, wohlwollende Haltung gegenüber dem Betriebsrat einzunehmen, umso eher ist Letzterer zur Verständigung, zur Beratung und zur Zusammenarbeit bereit (Hypothese H1).

(2) Je frühzeitiger das Management den Betriebsrat in die Entscheidungsprozesse der Organisation einbindet, umso stärker hat er den Eindruck, dass ihm Vertrauen entgegengebracht wird. Folglich wird dies die Bereitschaft des Betriebsrats zur konstruktiven Zusammenarbeit erhöhen (Hypothese $\mathrm{H} 2$ ). 
(3) Je stärker der Betriebsrat geneigt ist, eine offene, rücksichtsvolle Haltung gegenüber der Unternehmensleitung einzunehmen, umso fruchtbarer werden die gemeinsamen Beratungen sein (Hypothese $\mathrm{H} 3)$.

(4) Schließlich wird analog postuliert, dass das gegenseitige Verständnis positiv beeinflusst wird, wenn der Betriebsrat keinen Selbstzweck darin sieht, den Entscheidungsprozess zu verlangsamen. Dies wiederum wird den Unternehmenserfolg positiv beeinflussen (Hypothese H4).

Zusammengefasst gehen alle vier Hypothesen davon aus, dass eine konstruktive Haltung des Managements gegenüber dem Betriebsrat sich positiv auf die wirtschaftliche Lage des Unternehmens niederschlägt. Dennoch darf dieser Einfluss nicht verabsolutiert werden, d.h. auch die ökonomischen Ausgangsbedingungen des Unternehmens können relevant für das Agieren des Betriebsrats sein.

In unserer Analyse berücksichtigen wir deshalb auch die Frage, wie sich die Wirkungen der Mitbestimmung auf das Unternehmensergebnis verändern, wenn die Organisation restrukturiert werden muss. Cascio/Wynn (2004) vertreten die Auffassung, dass ein Restrukturierungsprozess nur dann effektiv gestaltet werden kann, wenn ein Arbeitsplatzabbau unter Mitwirkung der Arbeitnehmer vonstatten geht. Wenn die Beschäftigten in einen solchen Prozess nicht eingebunden werden, dann führt dies zu Passivität und Entmutigung zum Schaden der Organisation. Cascio und Wynn nennen vier Voraussetzungen für eine erfolgreiche Beteiligung der Mitarbeiter:

- frühzeitige Einbindung,

- eine sachkundige Belegschaft,

- Beratungen über Belange, die die Arbeitnehmer wirklich betreffen,

- eine Unternehmenskultur, die der Mitwirkung der Arbeitnehmer wohlwollend gegenübersteht.

Wenn diese Bedingungen erfüllt sind, dann werden die Arbeitnehmer motiviert bleiben und bereit sein, sich für die wirtschaftliche Erholung ihres Unternehmens einzusetzen. Diese Logik führt zu folgender Hypothese eines Interaktionseffekts (Hypothese H5): Wenn die Organisation ein Restrukturierungsprogramm durchläuft, dann wird der positive Einfluss einer gut funktionierenden Zusammenarbeit zwischen Unternehmensleitung und Betriebsrat (vgl. Hypothesen $\mathrm{H} 1, \mathrm{H} 2, \mathrm{H} 3$ und $\mathrm{H} 4$ ) auf die wirtschaftliche Lage des Unternehmens verstärkt.

\section{4 \\ Methodik}

Im Jahre 1998 wurde eine landesweite Studie in niederländischen Organisationen, die über einen Betriebsrat verfügen, durchgeführt. Ein umfassender Fragebogen wurde sowohl an das Management (Vorstand) als auch an die Betriebsräte von 3.500 Unternehmen, staatlichen Institutionen und anderen Organisationen geschickt. Die daraus resultierenden Daten wurden bislang nicht mit multivariaten ökonometrischen Methoden ausgewertet, sondern nur für bivariate Analysen genutzt. Für unsere hier vorgestellten Analysen verwenden wir ausschließlich die Daten der Umfragen unter den Unternehmensleitungen privater Unternehmen. Grund hierfür ist, dass bei den Befragungen nur die Vorstände gebeten worden sind, Angaben zur wirtschaftlichen Lage ihres Unternehmens zu machen, die wir für unsere Auswertungen benötigen. D.h. die Befragungen unter den Betriebsräten liefern zu diesem Punkt keine Erkenntnisse. Den öffentlichen Sektor haben wir deswegen ausgeschlossen, weil die dort Befragten häufig Schwierigkeiten hatten, ihre wirtschaftliche Situation genau einzuschätzen, sodass wir auch hier keine präzisen Ergebnisse erwarten konnten. Die schließlich verwendete Datenbasis umfasst 170 Privatunternehmen, für die alle relevanten bzw. uns interessierenden Informationen vorliegen. Im Gegensatz zu den verschiedenen Untersuchungen für Deutschland besteht unser Datensatz ausschließlich aus Organisationen, die einen Betriebsrat haben. Und anders als die meisten deutschen Analysen verfügen wir aus der Gesamterhebung über detaillierte Informationen darüber, wie die Unternehmensleitung und der Betriebsrat miteinander umgehen.

\subsection{ABHÄNGIGE VARIABLE: DIE WIRTSCHAFTLICHE LAGE DES UNTERNEHMENS}

Für die Frage nach der wirtschaftlichen Lage eines Unternehmes gibt es drei mög- liche Antwortkategorien: gesund/stark; etwas besorgniserregend; besorgniserregend/ schwach. Auf der Grundlage der Verteilung über die drei Kategorien haben wir beschlossen, die letzten beiden Kategorien zusammenzufassen. Für die Privatunternehmen haben $85 \%$ der Manager angegeben, dass das Unternehmen sich in einer gesunden/starken wirtschaftlichen Lage befindet. Van het Kaar/Looise (1999) haben die deskriptiven Statistiken aus diesem Datensatz mit ähnlichen Daten verglichen, die 1985 erhoben worden sind. Der Abgleich zeigt erwartungsgemäß einen deutlichen Unterschied zwischen beiden Erhebungszeitpunkten. 1998 haben die Manager die wirtschaftliche Lage positiver eingeschätzt als 1985. In der Tat waren die 1980er Jahre eine Krisenzeit mit Massenentlassungen und Unternehmenskonkursen, wohingegen die späten 1990er Jahre als wirtschaftliche Aufschwungphase gesehen worden sind, wobei sich erst später herausstellte, dass es sich dabei um die Internetblase handelte.

\subsection{KONTROLLVARIABLE: WIRTSCHAFTLICHE MESSGRÖßEN}

In Übereinstimmung mit den in der Literatur gängigen Annahmen über die Wirkungen der Betriebsratstätigkeit auf den Unternehmenserfolg enthält unser Basismodell zur Abbildung der wirtschaftlichen Unternehmenslage Charakteristika hinsichtlich Branche, Unternehmung, Belegschaft und Personalpolitik. Die branchenund firmenspezifischen Daten beziehen sich auf vier verschiedene Wirtschaftsbereiche, die Unternehmensgröße (sechs Kategorien), das Vorhandensein internationaler Verflechtungen und die rechtliche Eigenständigkeit. Die Charakteristika der Belegschaft betreffen den Anteil der festangestellten Arbeitnehmer, den Anteil der ungelernten Kräfte, die Erfahrung des Betriebsrats (auf einer Skala mit drei Stufen: in der Anfangsphase, ziemlich erfahren, professionell) und den Anteil von Teilzeitarbeit. Für die Charakteristika der Personalpolitik verwenden wir Informationen über das Personalmanagement (Human Resource Management (HRM)) und über die finanzielle Beteiligung leitender Angestellter. Insgesamt werden zwölf Meßgrößen des HRM unterschieden. Diese reichen von Mitarbeitergesprächen und Maßnahmen zur Stärkung des Teamgeists bis zu Weiterbildungsmöglichkeiten und Karriere- 


\begin{tabular}{|c|c|c|c|c|c|}
\hline Variablen für den privaten Sektor & $\begin{array}{l}\text { Mittel- } \\
\text { wert }\end{array}$ & $\begin{array}{l}\text { Standard- } \\
\text { abwei- } \\
\text { chung }\end{array}$ & Min & $\operatorname{Max}$ & $\chi^{2}$ \\
\hline Wirtschaftliche Lage & 0.847 & & 0 & 1 & \\
\hline Verarbeitendes Gewerbe & 0.424 & & 0 & 1 & $\chi^{2}(1)=0.18 \operatorname{Pr}=0.67$ \\
\hline Bauwirtschaft, Wohnungsbau & 0.135 & & 0 & 1 & $\chi^{2}(1)=0.89 \operatorname{Pr}=0.34$ \\
\hline $\begin{array}{l}\text { Verkehr, Handel, Dienstleistungen, } \\
\text { Gastgewerbe }\end{array}$ & 0.312 & & 0 & 1 & $\chi^{2}(1)=1.77 \operatorname{Pr}=0.18$ \\
\hline Banken und Versicherungen & 0.129 & & 0 & 1 & $\chi^{2}(1)=2.25 \operatorname{Pr}=0.13$ \\
\hline Unternehmensgröße & 3.259 & 1.51 & 1 & 6 & $\chi^{2}(5)=2.05 \operatorname{Pr}=0.84$ \\
\hline Internationale Zusammenarbeit & 0.406 & & 0 & 1 & $\chi^{2}(1)=2.38 \operatorname{Pr}=0.12$ \\
\hline Muttergesellschaft & 0.553 & & 0 & 1 & $\chi^{2}(1)=3.93 \operatorname{Pr}=0.05$ \\
\hline Festangestellte (in \%) & 87.364 & 8.79 & 60 & 100 & \\
\hline Ungelernte Kräfte (in \%) & 23.444 & 24.63 & 0 & 90 & \\
\hline Erfahrener Betriebsrat & 1.859 & 0.61 & 1 & 3 & $\chi^{2}(2)=6.30 \operatorname{Pr}=0.04$ \\
\hline Zunahme der Teilzeitbeschäftigten & 0.741 & & 0 & 1 & $\chi^{2}(1)=0.02 \operatorname{Pr}=0.90$ \\
\hline $\begin{array}{l}\text { Personalpolitische Maßnahmen } \\
\text { insgesamt (Summe der Werte) }\end{array}$ & 27.871 & 4.01 & 18 & 53 & \\
\hline $\begin{array}{l}\text { Gewinnbeteiligung von Führungs- } \\
\text { kräften (Summe der Werte) }\end{array}$ & 1.082 & 1.25 & 0 & 4 & $\chi^{2}(4)=7.30 \operatorname{Pr}=0.12$ \\
\hline Restrukturierung insgesamt & 0.424 & & 0 & 1 & $\chi^{2}(1)=11.87 \mathrm{Pr}=0.00$ \\
\hline Restrukturierung ohne Entlassungen & 0.253 & & 0 & 1 & $\chi^{2}(1)=0.08 \operatorname{Pr}=0.78$ \\
\hline Restrukturierung mit Entlassungen & 0.171 & & 0 & 1 & $\chi^{2}(1)=23.54 \operatorname{Pr}=0.00$ \\
\hline Haltung der Manager & 0.929 & & 0 & 1 & $\chi^{2}(1)=6.93 \operatorname{Pr}=0.01$ \\
\hline $\begin{array}{l}\text { Zeitpunkt der Einbindung des } \\
\text { Betriebsrats }\end{array}$ & 2.053 & 0.84 & 1 & 3 & $\chi^{2}(1)=5.73 \operatorname{Pr}=0.06$ \\
\hline Haltung des Betriebsrats & 0.718 & & 0 & 1 & $\chi^{2}(1)=13.14 \operatorname{Pr}=0.00$ \\
\hline Nicht verzögernder Betriebsrat & 0.424 & & 0 & 1 & $\chi^{2}(1)=0.18 \operatorname{Pr}=0.67$ \\
\hline \multicolumn{6}{|c|}{$\begin{array}{l}\text { Die letzte Spalte von Tabelle } 1 \text { zeigt die } \chi^{2} \text {-Statistik (und die dazugehörigen } p \text {-Werte) für die jeweilige Kreuztabelle der Dummy- } \\
\text { variablen für die wirtschaftliche Lage und aller unabhängigen Variablen. }\end{array}$} \\
\hline
\end{tabular}

aussichten. Die Daten zur finanziellen Beteiligung unterscheiden Gewinnbeteiligungen, Optionen, Aktien und Anleihen. Sowohl für das HRM als auch für die finanzielle Beteiligung haben wir zusammengesetzte Messgrößen konstruiert, indem wir die Werte für einzelne Charakteristika aufsummiert haben. Dabei spiegeln höhere Werte einen größeren Umfang von HRM und finanzieller Beteiligung wider.

Da der Schwerpunkt unserer Untersuchung auf der Wirkung der Mitbestimmung in Phasen des Arbeitsplatzabbaus liegt, verwenden wir detailliertere Variablen zur Abbildung der Restrukturierung. Von den Managern haben $42 \%$ berichtet, dass im Zeitraum von 1996 bis 1997 irgendeine Form der Restrukturierung stattgefunden hat. In $40 \%$ der Fälle war diese von Entlassungen begleitet. In der Basisanalyse unterscheiden wir zwei Typen der Restrukturierung: solche mit und solche ohne Entlassungen. Für eine sparsame Modellierung berücksichtigen wir bei der dritten Stufe unserer Schätzungen zur Analyse der Wirkung der Haltungen von Management und Betriebsrat in schlechten Zeiten nur, ob eine Restrukturierung stattgefunden hat oder nicht. ${ }^{2}$

\subsection{UNABHÄNGIGE VARIABLE: MAßE FÜR DIE MITBESTIMMUNG}

Auf Basis unserer Ausgangsüberlegungen (Abschnitt 2) unterscheiden wir im $\mathrm{Zu}$ sammenhang mit der Mitbestimmung vier verschiedene Aspekte. Wie die Manager ihre eigene Haltung beurteilen, wird in zwei Kategorien unterschieden: entweder als kompromissbereit und lösungsorientiert auf der einen Seite oder als formal, regelorientiert und machtbewusst auf der anderen Seite. Wie die Manager die Haltung des Betriebsrats wahrnehmen, unterliegt derselben Kategorisierung. Die Phase, in der der Betriebsrat in den Entscheidungsprowird auf einer dreistufigen Skala erfasst:

- der Betriebsrat überwacht nur die Umsetzung der Entscheidungen des Managements,

- der Betriebsrat hat in der Schlussphase des Entscheidungsprozesses ein Mitspracherecht,

- der Betriebsrat ist von Anfang an an den Entscheidungsprozessen beteiligt.

Eine Behinderung durch den Betriebsrat ist auf einer zweistufigen Skala erfasst: Hat das zess des Unternehmens einbezogen wird,
Management den Eindruck, dass der Betriebsrat den Entscheidungsprozess verlangsamt oder nicht?

Die Analyse der Wirkung der Mitbestimmung in Restrukturierungsphasen erfolgt durch Interaktionsvariablen, die durch Multiplikation der undifferenzierten Restrukturierungsvariable mit jeweils einer der vier Mitbestimmungsvariablen gebildet werden. Tabelle 1 zeigt die Daten der Untersuchungsbetriebe.

\subsection{SCHÄTZSTRATEGIE}

Wir beschreiben in der Folge die relevanten ökonometrischen Fragen, die unseren Schätzungen zugrunde liegen. Erstens ist bei den gegebenen Variablen für die wirtschaftliche Lage eine Probit-Analyse die angemessene ökonometrische Methode. Zweitens stellen wir hier nur die geschätzten Koeffizienten anstelle der üblicheren marginalen Effekte vor, (a) weil wir hauptsächlich an der Richtung und der Signifikanz der Effekte interessiert sind und (b), weil die Berücksichtigung von Interaktionsvariablen die Berechnung und Interpretation von Marginaleffekten erschwert. Drittens spiegeln infolge des Aufbaus der Umfrage alle Maße die Wahrnehmung von individuellen Befragten wider. Dies könnte zu zwei möglichen Problemen führen: gemeinsame Methodenvarianz (vgl. Podsakoff et al.2003) und Multicollinearität. Wir haben beides überprüft und sind $\mathrm{zu}$ dem Schluss gelangt, dass diese Probleme für die im Folgenden präsentierten Analysen keine Rolle spielen. ${ }^{3}$

\section{Empirische Befunde}

Tabelle 2 zeigt die Ergebnisse für die jeweiligen Spezifikationen. Unser Ziel ist es, zu untersuchen, ob die wirtschaftliche Lage einer Firma nur durch rein ökonomische Bestimmungsgründe erklärt wird oder viel-

\footnotetext{
2 Wir gestehen ein, dass die Schätzung eines simultanen Modells, das die wirtschaftliche Lage des Unternehmens und die Charakteristika der Mitbestimmung gemeinsam erklärt, vielleicht angemessener gewesen wäre.

3 Für weitere technische Einzelheiten siehe unsere ausführliche Vorstudie zu diesem Thema, die wir als Working Paper veröffentlicht haben (van den Berg et al. 2008).
} 
mehr auch durch die Ausgestaltung der Mitbestimmung und ihre Wahrnehmung. Folglich beginnen wir mit dem Basismodell, das nur die ökonomischen Kontrollvariablen enthält. Danach fügen wir unsere Variablen für die Mitbestimmung hinzu, was es uns ermöglicht, unsere ersten vier Hypothesen über die Haupteffekte zu testen. Im letzten Schritt der Analyse überprüfen wir unsere Hypothese des Interaktionseffekts. Dann untersuchen wir, ob die Effekte dieser Mitbestimmungsvariablen sich in schlechten (Restrukturierungs-) und in guten Zeiten unterscheiden und falls dies der Fall ist - in welchem Umfang.

\subsection{DAS BASISMODELL}

Spalte 1 in Tabelle 2 zeigt die Ergebnisse des Basismodells, bei dem die wirtschaftliche Lage eines Unternehmens durch die Branchen-, Firmen- und Belegschaftseigenschaften sowie durch die Ausprägung der Personalpolitik ${ }^{4}$ erklärt wird. Die Tabelle weist aus, dass Tochtergesellschaften schlechter abschneiden, dass Festangestellte sich in der Regel stärker für ihr Unternehmen einsetzen als befristet Beschäftigte und dass sich die Einführung umsichtiger Personalpolitik und finanzieller Anreize tatsächlich lohnt. Es gibt jedoch einen deutlichen Unterschied zwischen der Restrukturierung mit und ohne Entlassungen. Im ersten Fall sind die negativen Auswirkungen auf das Unternehmensergebnis deutlich stärker.

\subsection{DAS MITBESTIMMUNGSMODELL UNTER AUSSCHLIEßLICHER BERÜCKSICHTIGUNG DER HAUPTEFFEKTE}

In der zweiten Spalte von Tabelle 2 werden die Charakteristika bzw. Ausprägungen der Mitbestimmung hinzugefügt. Zusätzlich zum Basismodell stellen wir nun fest, dass größere und international orientierte Unternehmen sich tendenziell in einer besseren wirtschaftlichen Lage befinden. Außerdem wirkt sich der höhere Anteil festangestellter Mitarbeiter positiv aus, während eine Zunahme der Teilzeitbeschäftigung einen negativen Einfluss auf die wirtschaftliche Lage hat. Dies liegt aller Wahrscheinlichkeit nach an der allgemeinen Beobachtung, dass Menschen, die weniger Stunden arbeiten, gewöhnlich ein geringeres Engagement zeigen. Wir sehen außerdem, dass die Erfahrung des Betriebsrates nun das

\begin{tabular}{|c|c|c|c|}
\hline Variable & $\begin{array}{c}(1) \\
\text { Wirtschaftliche } \\
\text { Lage }\end{array}$ & $\begin{array}{c}(2) \\
\text { Wirtschaftliche } \\
\text { Lage }\end{array}$ & $\begin{array}{c}\text { (3) } \\
\text { Wirtschaftliche } \\
\text { Lage }\end{array}$ \\
\hline \multicolumn{4}{|l|}{ Unternehmens- und Brancheneigenschaften } \\
\hline Branchendummies & verwendet & verwendet & verwendet \\
\hline Unternehmensgröße & $\begin{array}{l}0.0731 \\
(0.60)\end{array}$ & $\begin{array}{l}0.292^{*} \\
(1.69)\end{array}$ & $\begin{array}{l}0.343 \\
(1.52)\end{array}$ \\
\hline Internationale Zusammenarbeit & $\begin{array}{l}0.617 \\
(1.64)\end{array}$ & $\begin{array}{c}1.098^{* *} \\
(2.19)\end{array}$ & $\begin{array}{l}1.412^{*} \\
(1.94)\end{array}$ \\
\hline Muttergesellschaft & $\begin{array}{l}-0.672^{*} \\
(1.87)\end{array}$ & $\begin{array}{l}-1.397 * * * \\
(2.81)\end{array}$ & $\begin{array}{l}-2.313^{* * *} \\
(2.96)\end{array}$ \\
\hline \multicolumn{4}{|l|}{ Eigenschaften der Belegschaft } \\
\hline Festangestellte - in \% - & $\begin{array}{c}0.0426^{* *} \\
(2.25)\end{array}$ & $\begin{array}{c}0.0609 * * \\
(2.33)\end{array}$ & $\begin{array}{l}0.108 * * * \\
(2.72)\end{array}$ \\
\hline Ungelernte Kräfte - in \% - & $\begin{array}{l}0.009 \\
(1.36)\end{array}$ & $\begin{array}{l}0.007 \\
(0.79)\end{array}$ & $\begin{array}{l}0.004 \\
(0.34)\end{array}$ \\
\hline Erfahrener Betriebsrat & $\begin{array}{l}-0.032 \\
(0.13)\end{array}$ & $\begin{array}{c}-1.111 * * \\
(-2.48)\end{array}$ & $\begin{array}{l}-1.847 * * \\
(2.58)\end{array}$ \\
\hline Zunahme der Teilzeitbeschäftigten & $\begin{array}{l}-0.332 \\
(0.90)\end{array}$ & $\begin{array}{c}-0.893^{* *} \\
(1.90)\end{array}$ & $\begin{array}{c}-1.568^{* *} \\
(2.44)\end{array}$ \\
\hline \multicolumn{4}{|l|}{ Charakteristika der Personalpolitik } \\
\hline Personalpolitik insgesamt (Summe der Werte) & $\begin{array}{l}0.115^{* *} \\
(2.49)\end{array}$ & $\begin{array}{l}0.125^{* *} \\
(2.34)\end{array}$ & $\begin{array}{l}0.224^{* *} \\
(2.35)\end{array}$ \\
\hline $\begin{array}{l}\text { Gewinnbeteiligung für Führungskräfte } \\
\text { (Summe der Werte) }\end{array}$ & $\begin{array}{l}0.333^{*} \\
(1.95)\end{array}$ & $\begin{array}{l}0.421^{*} \\
(1.87)\end{array}$ & $\begin{array}{l}1.152^{* *} \\
(2.53)\end{array}$ \\
\hline \multicolumn{4}{|l|}{ Arbeitsplatzabbau } \\
\hline Restrukturierung ohne Entlassungen & $\begin{array}{c}-0.960^{* *} \\
(-2.13)\end{array}$ & $\begin{array}{l}-1.422^{* *} \\
(2.18)\end{array}$ & \\
\hline Restrukturierung mit Entlassungen & $\begin{array}{l}-1.641 * * * \\
(4.14)\end{array}$ & $\begin{array}{l}-2.392 * * * \\
(4.25)\end{array}$ & \\
\hline Restrukturierung insgesamt & & & $\begin{array}{l}-10.04 * * \\
(2.50)\end{array}$ \\
\hline \multicolumn{4}{|l|}{ Charakteristika der Mitbestimmung } \\
\hline Haltung der Manager & & $\begin{array}{c}1.650^{* *} \\
(2.01)\end{array}$ & $\begin{array}{c}3.779 * * \\
(2.51)\end{array}$ \\
\hline Restrukturierung $x$ Haltung des Managements & & & $\begin{array}{l}2.750 \\
(1.36)\end{array}$ \\
\hline Zeitpunkt der Einbeziehung des Betriebsrats & & $\begin{array}{c}0.846 * * * \\
(2.66)\end{array}$ & $\begin{array}{l}0.698 \\
(1.31)\end{array}$ \\
\hline $\begin{array}{l}\text { Restrukturierung } x \text { Zeitpunkt der Einbeziehung } \\
\text { des Betriebsrats }\end{array}$ & & & $\begin{array}{c}2.288^{* *} \\
(1.99)\end{array}$ \\
\hline Haltung des Betriebsrats & & $\begin{array}{l}1.698^{* * *} \\
(2.95)\end{array}$ & $\begin{array}{l}3.917 * * * \\
(3.23)\end{array}$ \\
\hline Restrukturierung $x$ Haltung des Betriebsrats & & & $\begin{array}{l}-4.379 * * * \\
(3.01)\end{array}$ \\
\hline Keine Verzögerungen durch den Betriebsrat & & $\begin{array}{l}-0.444 \\
(-0.98)\end{array}$ & $\begin{array}{l}-3.063^{* *} \\
(2.53)\end{array}$ \\
\hline $\begin{array}{l}\text { Restrukturierung } x \text { keine Verzögerungen } \\
\text { durch den Betriebsrat }\end{array}$ & & (2.67) & $4.858 * * *$ \\
\hline Konstante & $\begin{array}{c}-5.600 * * * \\
(2.77)\end{array}$ & $\begin{array}{c}-7.034^{* *} \\
(2.52)\end{array}$ & $\begin{array}{l}-15.140 * * * \\
(2.95)\end{array}$ \\
\hline Zahl der Beobachtungen & 170 & 170 & 170 \\
\hline Mittelwert der abhängigen Variable & 0.85 & 0.85 & 0.85 \\
\hline Likelihood ratio & 54.94 & 82.04 & 97.91 \\
\hline Prob > chi2 & 0.000 & 0.000 & 0.000 \\
\hline Pseudo R² & 0.378 & 0.564 & 0.673 \\
\hline $\begin{array}{l}\text { Anmerkungen: } \\
t \text {-Statistik in Klammern; *** } p<0.01, * * p<0.05 \text {, and * } p< \\
\text { Die Mititestimmungsvariablen und ihhe interaktionsterme sind } \\
\text { Die vollständigen Schätzergebnisse sind auf Nachfrage erhältli } \\
\text { Quelle: Daten von van het Kaar/Looise (1999). }\end{array}$ & veils gemeinsam sign & WS & MITTEILUNGEN \\
\hline
\end{tabular}

4 Im Vergleich zu den in der Literatur üblicherweise verwendeten Variablen haben wir letztlich auf eine Analyse des Einflusses von Tarifverträgen verzichtet. Anfangs hatten wir in unseren Auswer- tungen eine entsprechende Variable berücksichtigt, es zeigte sich jedoch keinerlei Einfluss auf die Ergebnisse, sodass wir diese Variable schließlich ausgespart haben. 
Unternehmensergebnis in der Tat signifikant beeinflusst - jedoch negativ: Je erfahrener ein Betriebsrat ist, umso eher schwächt dies die wirtschaftliche Lage des Unternehmens. Dies könnte andeuten, dass diese Betriebsratsmitglieder ihre Erfahrung in der Praxis nicht zum Wohle der Firma nutzen, sondern sich in einem kontraproduktivem Gruppenverhalten festfahren, das konstruktive Beratungen eher behindert als anregt. Dieser Befund steht in engem Zusammenhang mit Theorien über Selektionsprozesse in Gruppen, die betonen, dass die Homogenität von Gruppen innerhalb von Organisationen tendenziell zunimmt, weil ihre Mitglieder dazu neigen, in einem Umfeld zu bleiben, das ihren Haltungen, Überzeugungen, Persönlichkeiten und Präferenzen entspricht (Boone et al. 2004). Wenn Menschen sich in einer geistesverwandten Gruppe befinden, dann bestärkt die lange Zugehörigkeit zu dieser Gruppe die Überzeugung, den richtigen Standpunkt zu vertreten, an dem sie dann folglich auch künftig festhalten. Lange Gruppenzugehörigkeit geht demnach mit Einheitsdenken und Trägheit einher.

Aus einer zweiten Beobachtung können wir schließen, dass die Art, wie die Mitbestimmung umgesetzt wird, in der Tat eine Rolle spielt. Insgesamt scheint die Mitbestimmung eine positive Wirkung auf die wirtschaftliche Lage von niederländischen Privatunternehmen zu haben. Erstens finden wir einen positiven Zusammenhang zwischen der Aufgeschlossenheit des Managements und der wirtschaftlichen Lage. Wenn der Vorstand eine wohlwollende Haltung gegenüber der Mitbestimmung hat, dann erhöht dies aller Wahrscheinlichkeit nach die Qualität der Beratungen und verbessert so indirekt das Unternehmensergebnis. Zweitens gilt analog, dass die frühzeitige Einbindung des Betriebsrats in die Entscheidungsprozesse des Unternehmens tendenziell das Vertrauen stärkt und es dem Betriebsrat somit ermöglicht, einen wertvollen Beitrag zur Unternehmenspolitik zu leisten, was sich dann in einem besseren Unternehmensergebnis niederschlägt. Drittens geht von einem Betriebsrat, der eine konstruktive Haltung zur Unternehmensleitung einnimmt, ein starker positiver Einfluss aus. Die positive Einstellung spiegelt wahrscheinlich die Tatsache wider, dass die Belegschaft ihre Vertreter in ihrer Kommunikation und Zusammenarbeit mit der Unternehmensleitung in betrieblichen Angelegenheiten unterstützt und sich dies für das Unternehmen günstig auswirkt.

Eine dritte Beobachtung liefert uns ein unerwartetes Ergebnis. Anstelle des angenommenen positiven Effekts eines Betriebsrats, der auf Verzögerungstaktiken verzichtet, ergab sich ein negativer, aber insignifikanter Einfluss. Wir werden diesen Punkt im folgenden Abschnitt noch einmal aufgreifen, weil der Befund sich ändert, sobald das Modell so angepasst ist, dass es eine Unterscheidung zwischen guten und schlechten Zeiten erlaubt.

\subsection{DAS ERWEITERTE MITBESTIMMUNGSMODELL MIT INTERAKTIONSTERMEN}

Zuletzt zeigt Spalte 3 in Tabelle 2, dass die Wirkungen der Mitbestimmung in Restrukturierungsphasen uneinheitlich sind. Dies schließen wir aus den Ergebnissen für die Interaktionsterme zwischen den Mitbestimmungscharakteristika und der allgemeinen Dummy-Variable für die Restrukturierung. Als Nebenergebnis haben wir festgestellt, dass der Einfluss all dieser verschiedenen Charakteristika auf die wirtschaftliche Lage des Unternehmens stärker wird, wenn wir diese Interaktionsvariablen in die Schätzung miteinbeziehen. Darüber hinaus beweist dies die Robustheit der Ergebnisse aus dem Basis- und dem Mitbestimmungsmodell. Der Haupteffekt aus (jedem der vier Charakteristika) der Mitbestimmung zeigt nun die Wirkung in guten Zeiten, ceteris paribus, und der Interaktionseffekt zeigt die Veränderung dieses Haupteffekts in schlechten Zeiten. ${ }^{5}$ Ein Blick auf die Vorzeichen sowohl der Hauptals auch der Interaktionseffekte verdeutlicht, dass sich die Wirkung der Mitbestimmung auf das Unternehmensergebnis in Phasen des Arbeitsplatzabbaus ändert. Wir werden jeden dieser signifikanten Effekte einzeln kommentieren.

Die positive Wirkung eines gegenüber dem Betriebsrat aufgeschlossenen Managements auf die wirtschaftliche Lage des Unternehmens wird in Phasen der Restrukturierung verstärkt. In schlechten Zeiten ergeht es dem Unternehmen erheblich besser, wenn das Management den Betriebsrat in konstruktiver Weise zu Rate zieht. Diese positive Wirkung der Mitbestimmung auf die wirtschaftliche Lage des Unternehmens in Umstrukturierungsphasen wird auch durch die Untersuchungsergebnisse für die Zeitplan-Variable bestätigt.
Je früher der Betriebsrat in den Entscheidungsprozess einbezogen wird, umso besser kann sich das Unternehmen wirtschaftlich behaupten. Auch dieser Effekt wird in Phasen des Arbeitsplatzabbaus verstärkt. Dieser Befund deckt sich mit unserer Hypothese und macht deutlich, dass es bei einer Restrukturierung günstig ist, den Betriebsrat möglichst früh hinzuzuziehen. Im Gegensatz zum positiven Einfluss eines aufgeschlossenen Managements scheint ein dem Management umgekehrt aufgeschlossen gegenüberstehender Betriebsrat die wirtschaftliche Situation eines Unternehmens während einer Restrukturierung unerwarteterweise negativ zu beeinflussen. Offensichtlich ist es von entscheidender Bedeutung, dass ein Betriebsrat in schlechten Zeiten stärker auf die formale Abwicklung der Restrukturierung achtet als nur darauf, ein angenehmer Gesprächspartner für das Management zu sein.

Das vierte Mitbestimmungscharakteristikum, ein Betriebsrat, der den Entscheidungsprozess nicht bremst, zeigt uneinheitliche Wirkungen. Der Haupteffekt wird signifikant negativ, aber ein zügig vorgehender Betriebsrat wirkt sich in schlechten Zeiten günstig auf das Unternehmensergebnis aus. Es scheint, dass die Umstände, unter denen ein Betriebsrat den Entscheidungsprozess lähmt, eine Rolle spielen. Folglich zeigt sich im Gegensatz zur Ausgangshypothese, dass es in guten Zeiten schädlich für die Unternehmensleistung ist, wenn der Betriebsrat den Entscheidungsprozess nicht bremst. Mit anderen Worten: Dies deutet an, dass ein Betriebsrat den Entscheidungsprozess in manchen Fällen nicht aus egoistischen Beweggründen verlangsamt, sondern weil er sorgfältiger vorgehen möchte. In schwierigen Zeiten möchte das Management jedoch schneller handeln. Dann behindert ein langsames Vorgehen des Betriebsrats den Entscheidungsprozess, was sich wirtschaftlich negativ auf das Unternehmen auswirkt.

Wenn wir die Ergebnisse aller drei Modelle betrachten, kommen wir zu dem Schluss, dass das Basismodell die erwarteten Zusammenhänge zwischen den wirt-

\footnotetext{
5 Aus Gründen einer sparsamen Modellierung fassen wir hier Restrukturierungen mit und ohne unfreiwilligen Arbeitsplatzabbau zusammen. Andernfalls hätten wir acht anstelle von vier Interaktionstermen erhalten. In Schätzungen, die wir hier nicht dokumentieren, haben wir nachgewiesen dass dies die zentralen Ergebnisse nicht verändert.
} 
schaftlichen Charakteristika und der wirtschaftlichen Lage nachweist. Aus dem Mitbestimmungsmodell können wir folgern, dass die Art, wie die Mitbestimmung im Unternehmen umgesetzt wird, das Unternehmensergebnis in gleicher Richtung beeinflusst. Wenn wir das Mitbestimmungsmodell erweitern, um festzustellen, ob die Mitwirkung der Arbeitnehmer die wirtschaftliche Lage des Unternehmens in Restrukturierungsphasen verbessert oder verschlechtert, dann kommen wir zu einem bemerkenswerten Ergebnis. Einerseits wirkt sich die Mitbestimmung erwartungsgemäß positiv auf den wirtschaftlichen Erfolg des Unternehmens aus, wenn das Management bereit ist, die Arbeitnehmervertreter ernstzunehmen und sie durch seine positive Einstellung und die frühzeitige Einbindung gewinnt. Andererseits wird die Unternehmensleistung in Phasen des Arbeitsplatzabbaus überraschenderweise negativ beeinflusst, wenn die Arbeitnehmervertreter infolge ihrer positiven Grundeinstellung bereit sind, konstruktiv mit dem Management zusammenzuarbeiten. Außerdem stellen wir im Gegensatz zu unseren Erwartungen fest, dass ein Betriebsrat, der den Entscheidungsprozess nicht verlangsamt, der Organisation in guten Zeiten schadet.

\section{Fazit}

In ihrer bahnbrechenden Arbeit zur Ökonomie der Mitbestimmung vertreten Freeman/Lazear (1995) die Ansicht, dass ver- schiedene Mitbestimmungsrechte Arbeitnehmer dazu bringen können, zum Wohle des gesamten Unternehmens mit dem Management zusammenzuarbeiten. Die Autoren verweisen aber auch auf mögliche Schattenseiten der Mitbestimmung, da diese eine Reihe zusätzlicher Kosten einschließlich eines Rent-Seeking-Verhaltens verursachen kann. Bryson et al. (2006) entwickeln dieses Argument weiter und vertreten die Auffassung, dass die Mitsprache der Arbeitnehmer nur erfolgreich funktionieren kann, wenn sie vom Management unterstützt wird.

Unser Hauptergebnis ist, dass die Art, wie das Management und die Betriebsräte miteinander umgehen, die wirtschaftliche Lage einer Organisation entscheidend beeinflusst. Zuerst wird das „britische Ergebnis“ von Bryson et al. (2006) für die Niederlande bestätigt: Eine positive Einstellung der Manager gegenüber dem Betriebsrat ist positiv mit dem Unternehmenserfolg verbunden. Dieses Ergebnis ist in Phasen der Restrukturierung noch ausgeprägter. Außerdem stellen wir fest, dass es sich ebenfalls positiv auswirkt, wenn die Unternehmensleitung den Betriebsrat frühzeitig in den Entscheidungsprozess einbindet. Wieder wird dieser positive Effekt in Phasen des Arbeitsplatzabbaus verstärkt.

Die Einstellung der Arbeitnehmervertreter spielt ebenfalls eine wichtige Rolle. Wir finden einen großen Unterschied zwischen ihrem Einfluss in wirtschaftlich guten und in schlechten Zeiten. In guten Zeiten korreliert ein aufgeschlossener Betriebsrat positiv mit dem Unternehmens- erfolg, in schlechten Zeiten hingegen ist dieser Zusammenhang negativ, was nahelegt, dass in Restrukturierungsphasen eine formalere Haltung der Arbeitnehmervertreter besser für die Organisation sein kann. Außerdem wirkt sich ein langsam arbeitender Betriebsrat in guten Zeiten günstig aus, vermutlich, weil ein solcher Betriebsrat stärker auf eine sorgfältige Entscheidungsfindung achtet. Diese Eigenschaft des Betriebsrats schädigt das Unternehmen jedoch in wirtschaftlich schwierigen Zeiten.

Wie wir einleitend erwähnt haben, stammen unsere Daten von der (beschreibenden) Studie von van het Kaar/Looise (1999). Sie folgern in ihrem Schlusskapitel (S. 257-259), dass die niederländischen Betriebsräte in den 1990er Jahren einen Reifeprozess durchlaufen haben. In den Bereichen Personal- und Organisationspolitik sind sie zu anerkannten Gesprächspartnern avanciert. Begrenzt bleibt ihr Einfluss auf geschäftliche, finanzielle und technologische Entscheidungen. Nur eine Minderheit von Unternehmensleitungen erlaubt ihren Betriebsräten, in diesen Bereichen mitzuwirken. Unsere ökonometrischen Forschungsergebnisse lassen sehr stark vermuten, dass die Wirksamkeit der Betriebsratstätigkeit hinsichtlich des Unternehmenserfolgs in den Niederlanden in wirtschaftlich guten wie schlechten Zeiten noch weiter gestärkt werden könnte, wenn die Mehrheit der Manager bereit wäre, die Arbeitnehmervertreter sowohl frühzeitig als vollwertige Partner einzubinden, als sie auch stärker als bisher in strategische Überlegungen einzubeziehen. 
Addison, J. T. (2005): The Determinants of Firm Performance: Unions, Works Councils and Employee Involvement. High Performance Work Practices, in: Scottish Journal of Political Economy 52, S. 406-450 Addison, J.T./Bellmann, L./Schnabel, C./Wagner, J. (2004): The Longawaited Reform of the German Works Constitution Act, in: Industrial Relations 43, S. 392-420

Addison, J. T./Schnabel, C./Wagner, J. (2001): Works Councils in Germany: Their Effects on Establishment Performance, in: Oxford Economic Papers 53, S. 659-694

Addison, J. T./Schnabel, C./Wagner, J. (2004): The Course of Research into the Economic Consequences of German Works Councils, in: British Journal of Industrial Relations 42, S. 255-281

Boone, C./Van Olffen, W./Van Witteloostuijn, A./De Brabander, B. (2004): The Genesis of Top Management Team Diversity: Selective Turnover among Top Management Teams in the Dutch Newspaper Publishing, 1970-94, in: Academy of Management Journal 47, S. 633-656 Bryson, A./Charlwood, A./Forth, J. (2006): Worker Voice, Managerial Response and Labour Productivity: An Empirical Investigation, in: Industrial Relations Journal 37, S. 438-455

Cascio, W. F./Wynn, P. (2004): Managing a Downsizing Process, in: Human Resource Management 43, S. 425-436

Dilger, A. (2002): Ökonomik betrieblicher Mitbestimmung. Die wirtschaftlichen Folgen von Betriebsräten, München und Mering
Freeman, R. B./Lazear, E. P. (1995): An Econometric Analysis of Works Councils, in: Rogers, J./Streeck, W. (Hrsg.): Works Councils - Consultation, Representation and Cooperation in Industrial Relations, Chicago Freeman, R. B./Medoff, J. L. (1984): What Do Unions Do?, New York Looise, J. C./Drucker, M. (2003): Dutch Works Councils in Times of Transition: The Effects of Changes in Society, Organizations and Work on the Position of Works Councils', in: Economic and Industrial Democracy 24, S. 379-409

Podsakoff, P. M./MacKenzie, S. B./Lee, J.-Y./Podsakoff, N. P. (2003): Common Method Biases in Behavioral Research: A Critical Review of the Literature and Recommended Remedies, in: Journal of Applied Psychology 88, S. 879-903

Van den Berg, A./Grift, Y./Van Witteloostuijn, A. (2008): Works councils and organizational performance. The role of top managers' and works councils' attitudes in bad vis-à-vis good times, Diskussionspapier Tjalling C. Koopmans Institute 08-14

Van het Kaar, R. H./Looise, J. C. (1999): De volwassen OR: groei en grenzen van de Nederlandse ondernemingsraad. Resultaten van het grote OR-onderzoek (Der mündige Betriebsrat: Entwicklung und Grenzen des niederländischen Betriebsrats. Ergebnisse der großen Betriebsratsstudie), Alphen aan den Rijn 